\title{
WEB 2.0 Pharmacy Robots
}

\author{
doi:10.3991/ijoe.v6s1.1389 \\ J. García Zubia, I. Trueba Parra , D. Lopez-de-Ipina \\ University of Deusto, Bilbao, Spain
}

\begin{abstract}
This paper presents a new generation of pharmacy robots based on micro-servers and web 2.0 technologies. Micro-servers make accessibility and remote configuration of the robot easier, because it does it by native way. This new technology offers a faster, more robust and ecologic service than the classic solutions. A robotic prototype has been built, installed and checked successfully. This fact presents a new paradigm between classic architecture and this new one in the robots control area.
\end{abstract}

Index Terms-Micro-servers, pharmacy, remote control and robots.

\section{INTRODUCTION}

Daily life in the pharmacy is very hectic, dealing with costumers, searching the requested medicaments, preparing new orders, receiving them, and so on. This paper presents a solution to dispense the medicaments, and load the received orders. So, on the one hand, the life of the pharmacist is going to be easier and more comfortable. On the other hand, the pharmacists will have the opportunity to improve the service quality, offering a closer and attentive deal with costumers.

Until now, the process automation was only available to multinational companies; however, the market is tending to automate their facilities due to their enormous advantages [1].

Today, all sectors tend to automate their processes to increase performance, reduce costs and to offer faster and more satisfactory service to customers, which are increasingly enhancing their expectations. The pharmaceutical sector is one of them [2].

Pharmacy robots would facilitate the daily work of the pharmacist, who wouldn't have to worry about searching each medicament and allowing him to offer a better focus on each customer [3]. In this way, the automated pharmacy becomes a much more dynamic pharmacy than the classic one, where customers demand flows faster and with the satisfaction of receiving a better personal approach. [4]:

Some other advantages of automated pharmacies are

- Optimization of time

- Improve the use of physical space

- Stock management automatically

Automated pharmacies are equipped with a dispenser robot, which communicates with the PC of the pharmacist waiting to new product orders

Automated pharmacies have a dispensing robot that is in communication with the PC pharmacist pending new product orders. When the pharmacist sends the order, the

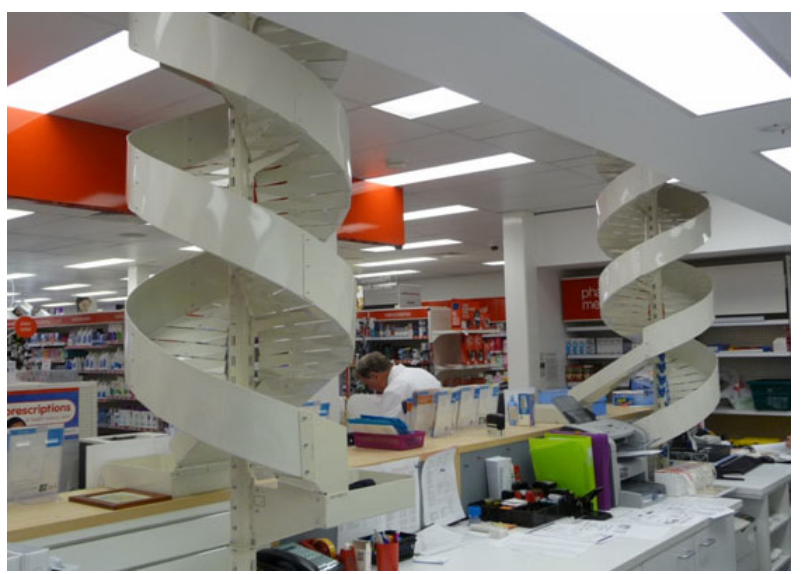

Figure 1. Automated pharmacy [5]

robot dispenses the product automatically and transports it to the pharmacy counter (Fig. ${ }^{\circ} 1$ ).

This paper aims to provide an overview from all the classical solutions [6] available related with pharmacy automation and to present a new generation of robots, based on Web 2.0 technologies [7] micro-servers and a novel control architecture [8] with the purpose of revolutionize the sector because of its high performance/cost ratio.

\section{Classical SOluTiONS}

The automation of pharmacies is a concept which has been developed since the early 90s. Nowadays, there are different dispensing robots types, depending on the number of kind of products that want to automate and the rotation of these products.

It may be classified as [9]:

- Few kind of products, high rotation

- Many kind of products, low rotation

- Hybrid System

Robots dispensers with high rotation and few kind of products ( $<250$ kind of products) are characterized by being composed by a slanted shelves, which store different types of products separated by small profiles (Fig. 2). The robot is loaded from its back (the top of the shelves), while the dispensing is done by the front, by a manipulator which lies on two linear units (x-axis and $\mathrm{z}$ axis) that provide it mobility across the width and height of the robot. This manipulator picks up one by one the ordered products and leaves them on the conveyor belt, so, it has to be moved at high speed to be able to deliver successfully all the orders (which requires a large number of movements due to its architecture), in acceptable times. Due to the high speeds reached by the manipulator, it is necessary to enclose the robot, for safety reasons, thus preventing ac- 


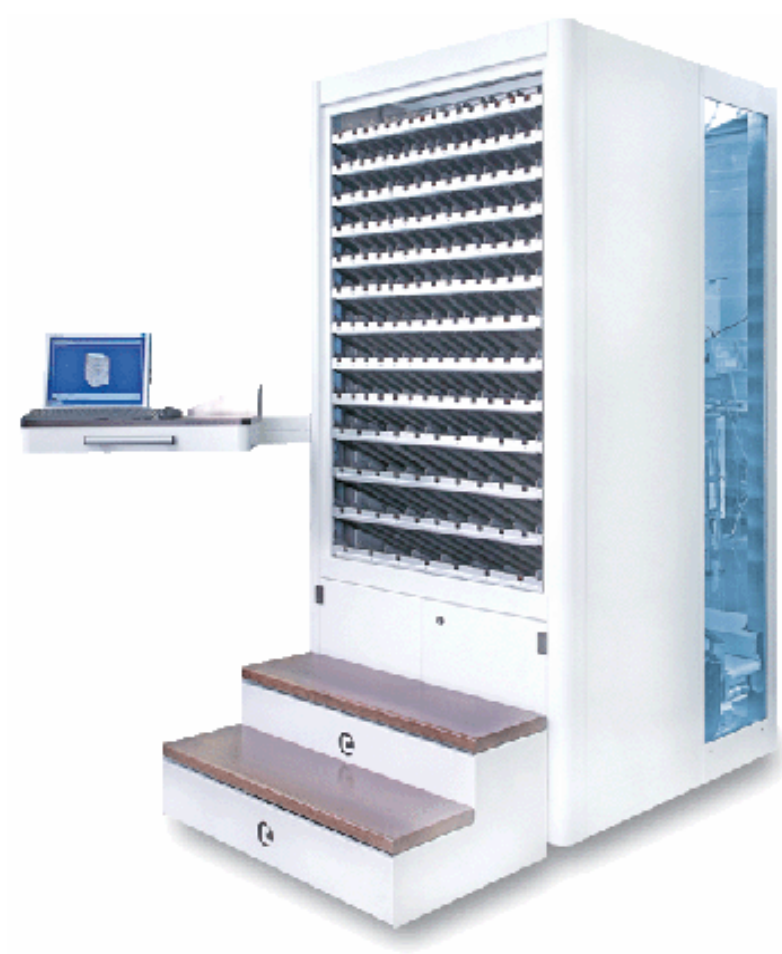

Figure 2. Dispensing robot manufactured by ROWA[10]

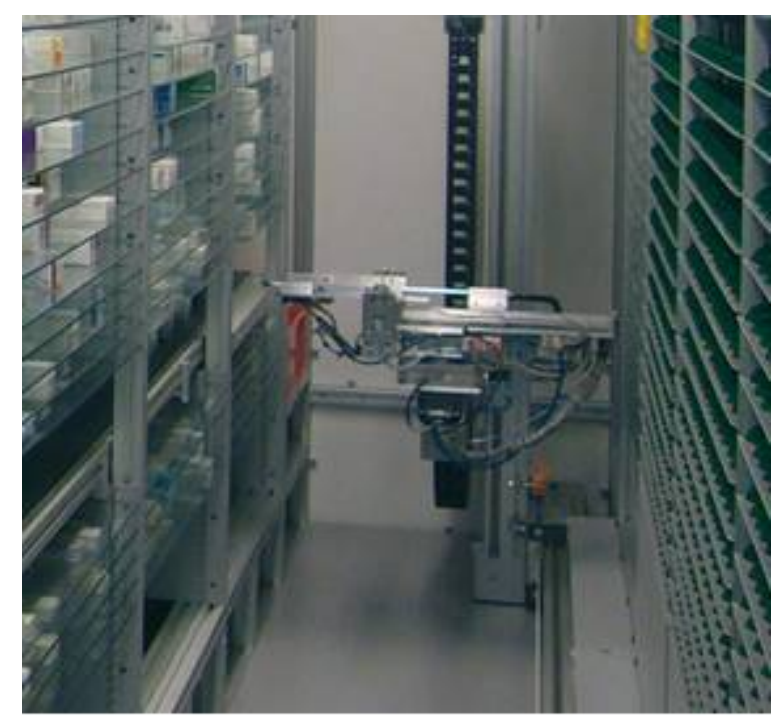

Figure 3. Tecnifarma Robot [11]

cess to anyone to avoid possible accidents, so that, in case of breakdown or failure, the pharmacist is unable to access the medications comfortably, which can cause a temporary interruption of the pharmacy service.

Thanks to the arrangement of the shelves cited above, the first product stored in the dispensing robot, will be the first product to be dispensed, creating a FIFO (First-InFirst-Out), avoiding problems of expiry. As mentioned above, the robot is facing a conveyor belt whose target is to collect the medicaments dispensed by the robot and to transport them to the counter.

Meanwhile, low rotation systems and many kind of products, also called chaotic, are a totally different concept to that described above (Fig. 3). In this case, the medicaments do not have a predefined storage location as before.

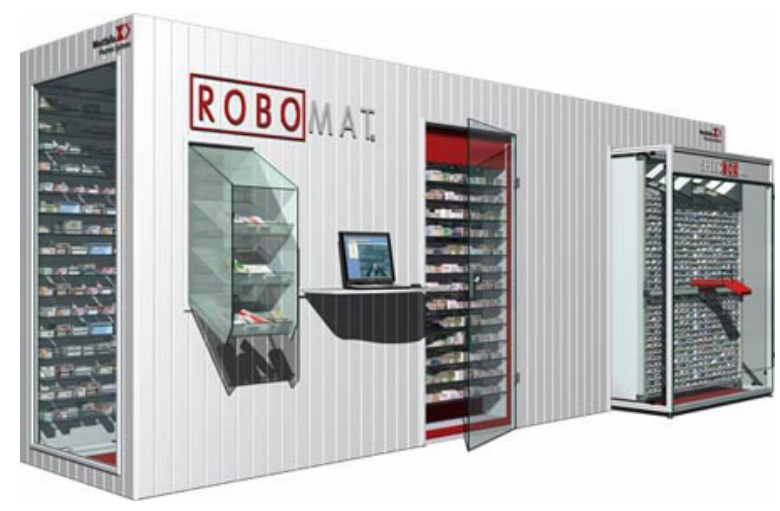

Figure 4. Hybrid Robot by Match4[12]

Now, a manipulator is in charge to store medication in any warehouse location, recording its position in its database for subsequent dispensing. The most widespread system consists on horizontal shelves where the products are deposited by a manipulator equipped with tweezers.

A pharmacy is composed by medicines of high and low rotation, so that, hybrid robots have appeared (Fig. 4). This robot type allows the storaging of products massively (chaotic system) and the storage of high rotation products. As in the rest of the classical solutions seen so far, hybrid robots are based on manipulators, so they have the disadvantages or problems described in previous paragraphs.

These robots have been the first step towards the automation of the pharmacies. Their functionality make the pharmacist' life easier; however, there are many things to improve (speed, integration, architecture, installation, technology and so on). This proposal solution improves many of this items without forgot the final target, make the pharmacist life easier.

\section{PROPOSED SOLUTION}

Before presenting the new solution for pharmacy robots it is essential to present the micro-servers. There is no doubt that they are the devices that make the difference and allow the leap to a new generation of robots in pharmacy.

\section{A. Micro-servers}

The micro-servers are devices which are equipped with a specific electronic that is in charge to communicate with the main server and execute commands. This kind of device is very widespread in the industrial area. Strong companies, like Siemens [13], develop them. Particularly, the device presented in this paper has incorporated a mechanical system to the micro-server electronic, which allows it to dispense a medicament by itself (Fig. 5).

The main element of this new control architecture, which will be presented in this paper, is the micro-server. At first time, the University of Deusto began to work with this device to create Web-Labs [14]. The purpose of these virtual laboratories is academic. These novel laboratories are one step closer to integration of Web services of the university as a base to train students. Thanks to the WebLabs, many students can connect their PC to the same equipment remotely. The micro-server is responsible for managing the timing and controlling the assigned equipment to the student. It requests the service through its control chip, which is the element that brings intelligence 


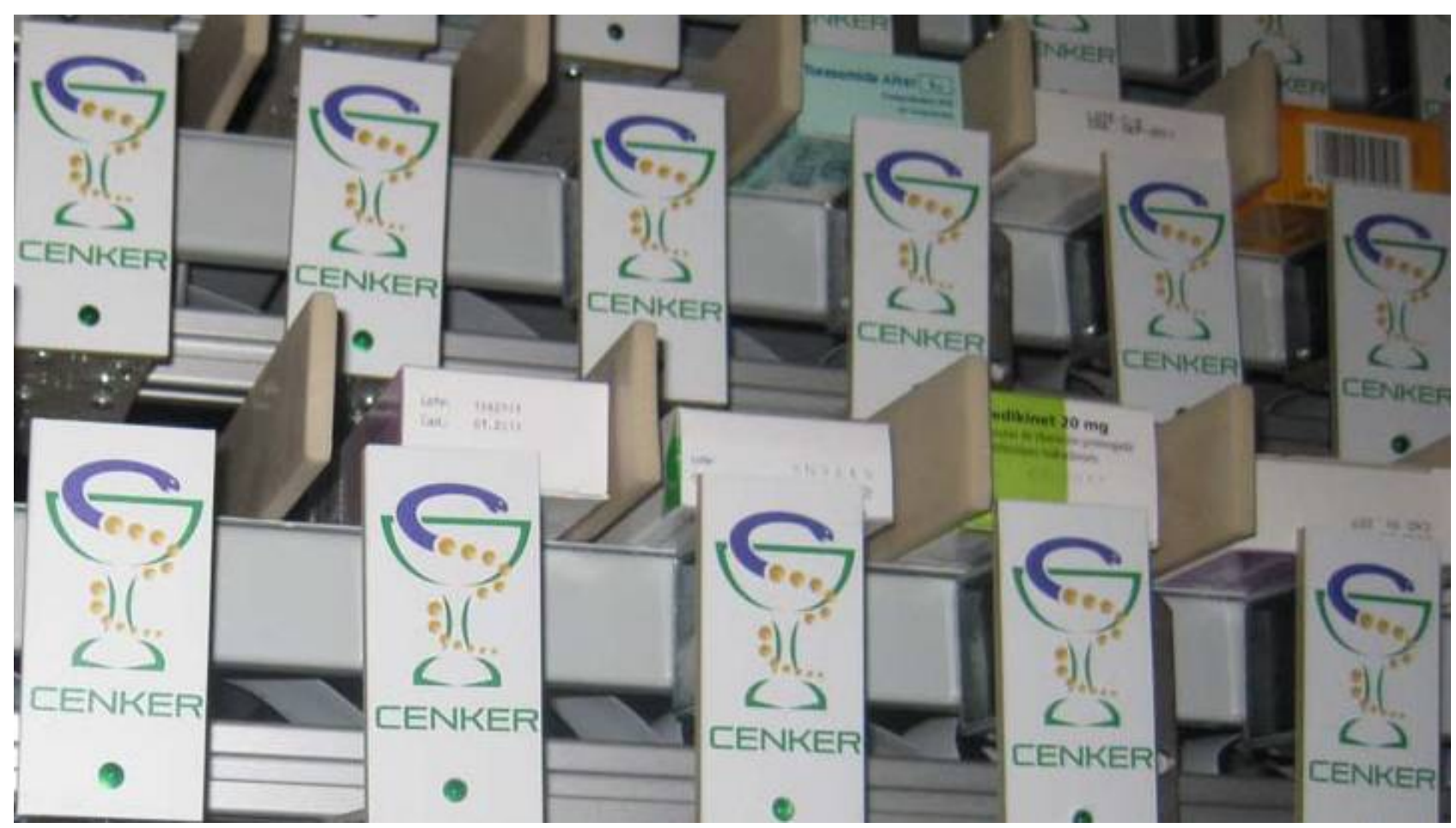

Figure 5. Front view of microservers used in the robot

to the device. With this new type of laboratory, the physical concept disappears, as the student can make their training exercises from anywhere and at any time and for this reason the restrictions that the classic laboratories schedules disappear. A single requirement: Internet connection.

Thanks to these Web-Labs, the University realized how many opportunities these simple but powerfull devices have. For this reason, it decided to develop these microservers towards the industrial world. They are much more robust thanks to their industrialization and have gone deeper into service management area. This new version of micro-servers incorporates a mechanical system able to deliver the medication required by the system. Infrared sensors, installed at strategic points of the device, check product stocks and the correct supply of the product. Then, the micro-server sends the results of the checking to the main server. These functionalities make this device something superior and something not seen before. In fact, this device is protected by an international patent

The micro-servers use means the control decentralization, providing intelligence to the field sensors/actuators, which is a general trend in all sectors since the last decade. With this new device, will be increased the performance and the system will be simplified.

\section{B. Web 2.0 technology}

The architecture of these dispensing robots is based on micro-servers (Fig. 6). The micro-servers provide all field devices with local intelligence. This induces the decentralization of the control system, which, with the help of new technologies, allows full interactivity among pharmaceutical and the customers and in turn between pharmacist and pharmaceutical warehouse, which improves the system and makes it easier to work daily to pharmacists because the mistakes are minimized.

The architecture of this new delivery system is based on advanced technologies which are leaders, as those imple-

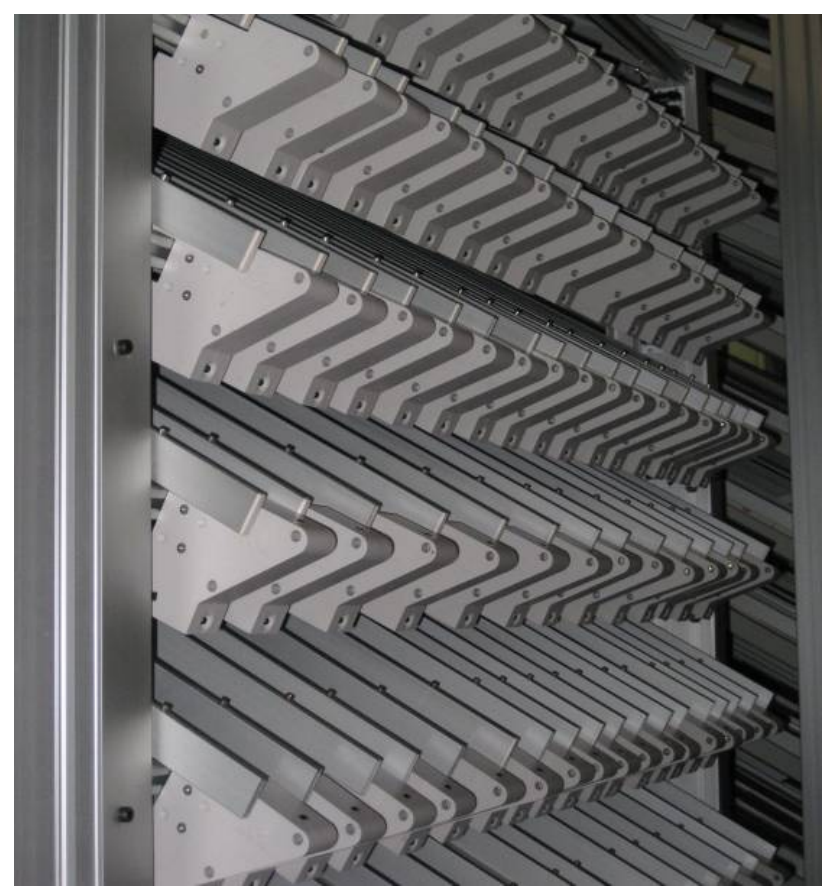

Figure 6. Perspective of micro-server

mented under free software. These technologies offerpower, speed, the possibility to create friendly environments for the end user and quality. In addition, everything without the expensive cost of licenses or other fees which makes the product price higher. In this case, the control architecture is built on web technology, which adds an additional dimension to the system. A database based on the MySql engine and control algorithms in PHP programming language are responsible for supporting the system control. These two elements were originally created for websites. 


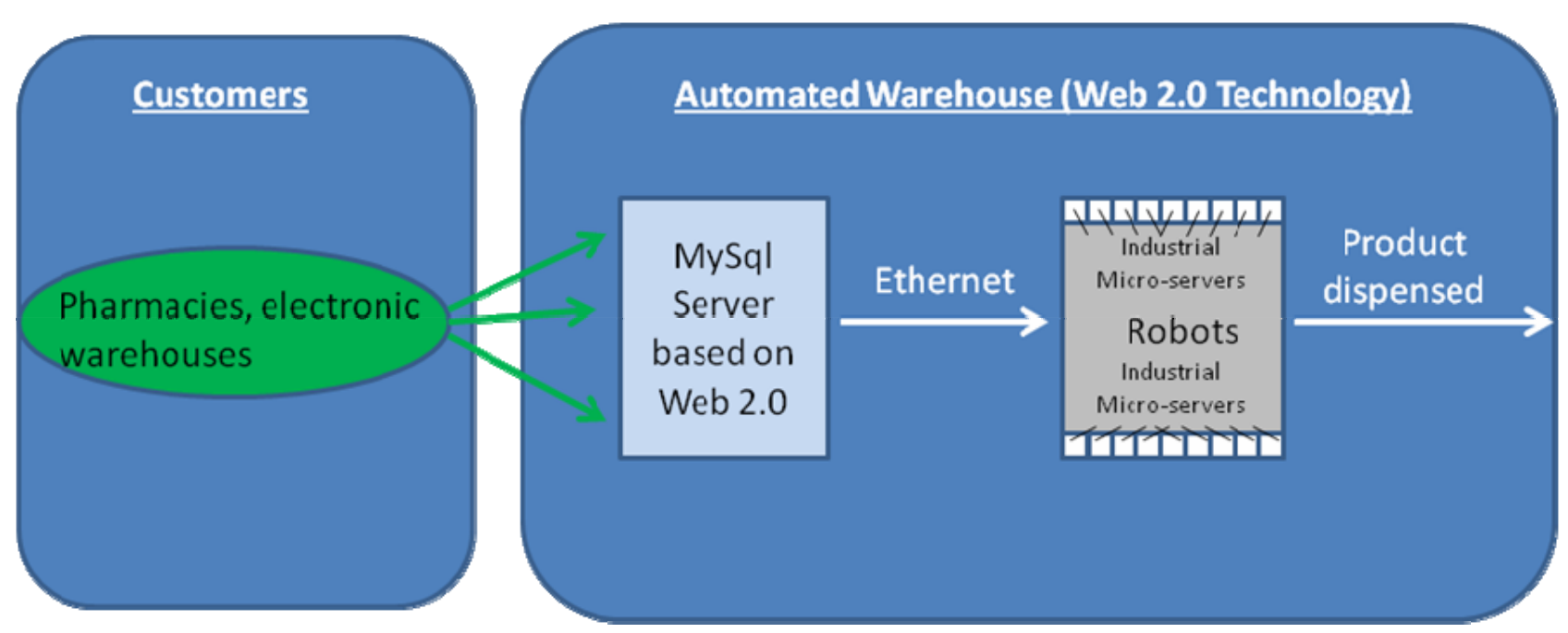

Figure 7. Control architecture based on Web 2.0 technology

However, their higher performance and simple way of operating, are allowing them to increase the number of sectors where they are installed. So, when the pharmacist asks for a product to the system, it gives the order to the corresponding micro-server and, after checking the availability of the product, it dispenses the medicament through a mechanical device. If the product requested is not available or has not been supplied, the micro-server will detect it, generating an alarm in real time.

In fact, considering market trends, the use of this web technology is a great decision as it will allow in the near future (when the pharmaceutical sector will realize about the reliability and working capacity of these robots, eliminating any skepticism), the interaction between the pharmacy and the central warehouse. In this case, the pharmacy system would be the one which would arrange automatically the orders for the central warehouse, in the moment it detects that there is the necessity to increase the stock. Thus, pharmacists should only need to focus their efforts on serving customers and increasing their profits as much as possible.

Thanks to web technology, the user has access to the system from anywhere where there was Internet access and he will able to know in real time the status of the installation and to get any information needed.

Moreover, the micro-servers not only have been useful in dispensing, but also have been used in the arduous task of replacing the new medicaments in the robot: semiautomatic repositioning.

Before semi-automatic repositioning, pharmacists loaded the robot manually with the help of long lists that tells them, which was the location of each product in the robot. This task was a big waste of time. With semiautomatic repositioning, the task is performed with greater fluency and clearly increasing the performance of the pharmacy.

The device consists on a card with some LEDs that are placed on top of each shelf (Fig. 8). These cards are equipped with micro-servers that are responsible for communicating with the system and receiving their orders. Moreover, the cards are designed to make possible the interconnection of multiple cards with each other without wires. The operation of the system is simple: the user introduces the product of the medicament that he wants to

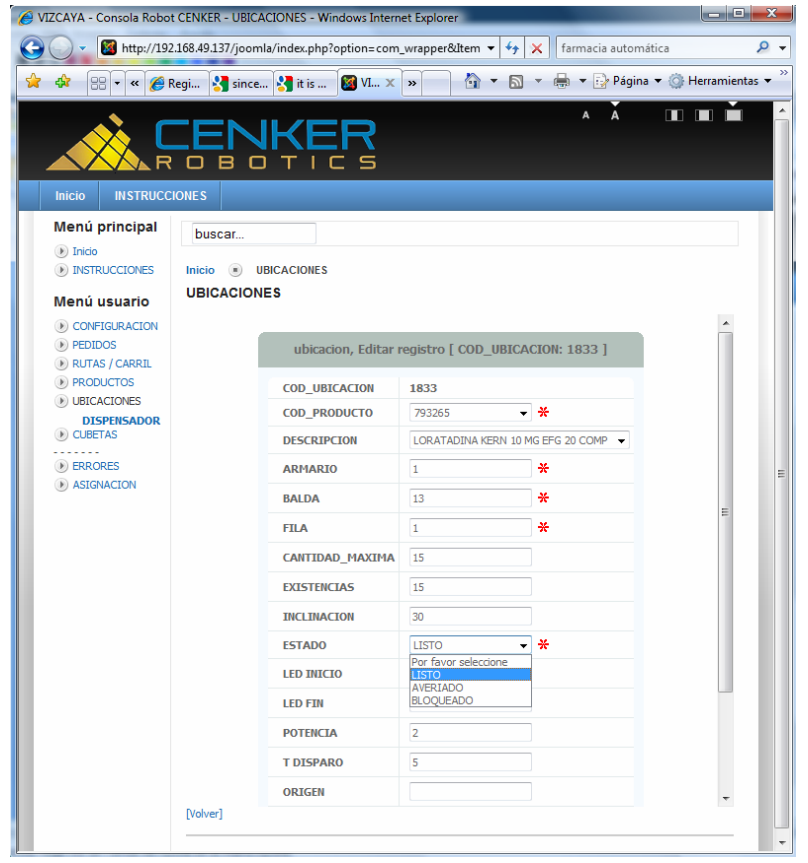

Figure 8. Web server making changes

load, via PC or PDA and the system communicates with the corresponding micro-server by illuminating the LED's where the drug has to be introduced.

Thanks to this technology, the pharmacist can load a large number of products in a short time with a single glance. This makes their daily tasks easier and reduces significantly the time required for the medicaments replenishment.

\section{Remote control}

The architecture used during the design of these novel robots allows a great versatility, real-time versatility. The system can be configured by remote way because all the system is managed by an architecture based on web 2.0 technology and all the devices, which take part of this architecture, have intelligence. A Web server (Fig. 8) allows changing the parameter of the robots. These changes save on MySQL database in real-time. So, the pharmacist can make changes in his installation, wherever he was. 


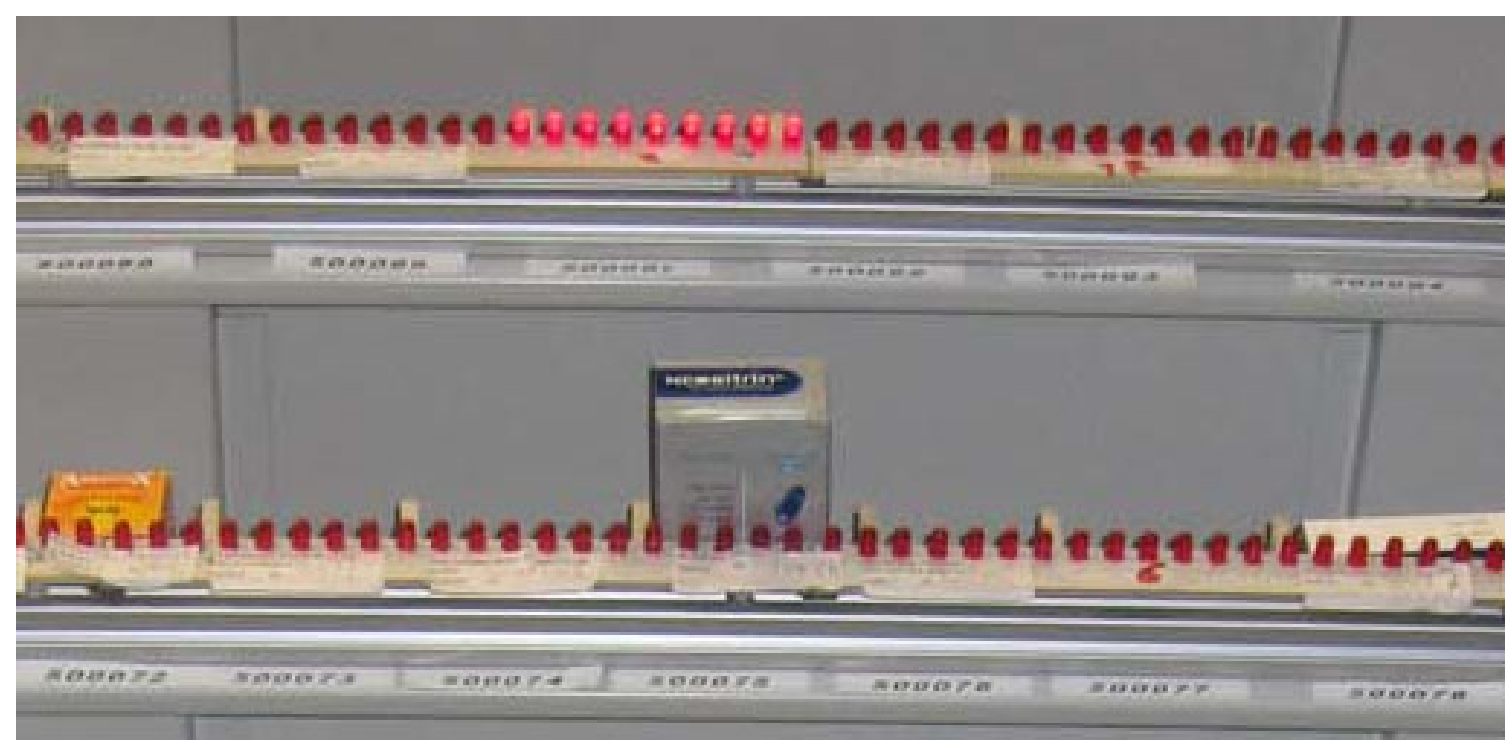

Figure 9. Rear part of robots with led Cards based on micro-servers

With this new architecture, the system functionality increases a lot. The system configuration is only a simple example to show the power of the system, but with this technology, the user can connect with the system remotely and ask it all the information about the state of the installation or whatever. In the same way, the pharmacist can connect to the Web server and ask all the needed information. The options and functionalities of the system can be customized, depending on the customer needs.

This system, and thanks to the new technologies, removes any kind of spatial-temporal obstacle because on the one hand, the user is going to be able to connect to the system wherever he is (with an internet connection). On the other hand, the user could ask or search any information whenever he needs it.

Obviously, most of the maintenance of the system is going to make remotely, avoiding operational interruption and improving the service. It is possible to know the status of the installation by Web way, so the maintenance and alarms control can be solved and managed by this way: Web way.

These three items are the key of the new solution presented on this paper. Furthermore, the instructions to use it are very simples, so pharmacist will feel comfortable since the first day. When a customer asks a product, the pharmacist sends the order by the PC software. This software runs on PHP.

It communicates the order to the MySQL database and, thanks to the WEB 2.0 architecture; it sends the order to the micro-servers too. The micro-servers dispense the order and they communicate their movements to MySQL database. Finally, the pharmacist software receives a report about the order. This report is visualized by the pharmacist software. As you can see, the process is transparent and easy for the pharmacist. As it can see, the remote control is a characteristic very important in the system, providing it with a lot of functions which have ever seen before.

\section{AppliCAtion OF THE New Solution}

It's time to present the new pharmacy robot that is able to dispense and help to load medications (Fig. 9). This new robot, based on micro-servers and Web technology, is modular, avoiding mega-installations.

Each module consists of a metal frame completely customizable to customer requirements and dimensional restrictions of the own pharmacy. This versatility is unique in the market. These frames contain inclined shelves where products are stored, so the first product, which is loaded on shelve, is the first product dispensed by the robot; each type of product is separated by small profile. Once products are inserted into the dispensing robot, they are supported by its own micro-server, which, thanks to its particular ergonomic, also acts as a stop point for products.

When the user requests a order to the system, this dispenses the medicament on conveyor belt that is in charge to carrying it to the pharmacy counter. Thanks to the micro-servers and the control architecture used is possible to dispense simultaneously so many medicaments as kind of products have been stored, which means a dispensing speed much higher than any other classical solution.

Until now, it has not been mentioned another strong point of the system: the automatic repositioning of medicines. This system has the ability to load automatically the new medicaments.

The automatic repositioning system is able to load automatically the medicaments from one or more entry points (depending on customer needs). After inserting the products in the repositioning system, this is the responsible for transporting and directing each product to its corresponding dispenser through a cyclic transport system. Once the product reaches its dispenser, other electronic device is responsible for verifying the identity of the product and storing it in its corresponding position.

This dispensing robot is specially designed for pharmacies, and thanks to its size and modularity can be installed on any type of pharmacy, whatever its size and shape.

\section{ANALYSIS OF THE SOLUTION}

The new generation of pharmacy robots is already presented, so it is the appropriate moment to review the advantages of this new technology in the pharmacies and compare them with the classic solutions used until now. 


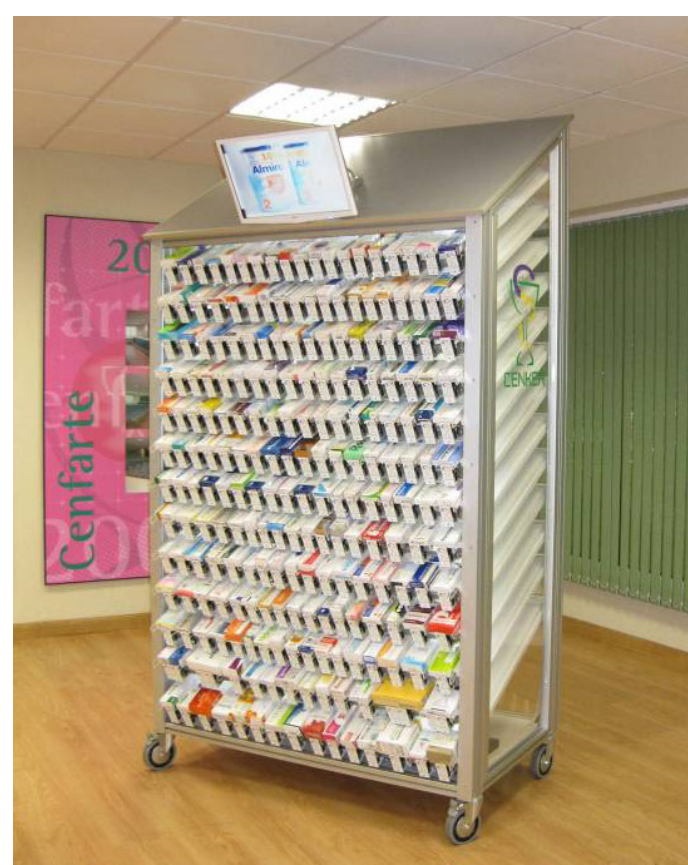

Figure 10. New pharmacy robot based on micro-servers

The installation of these robots is easy and fast (never more than a week) avoiding any kind of discomfort. Until now, the pharmacies had to adapt to the robot; now, with this architecture and technology, the robot adapts to the pharmacy. This is possible because the robot is a lightweight, easy to carry and fully modular. A classical robot weighs around $500 \mathrm{~kg} / \mathrm{m}^{2}$ meanwhile, the new solution don't weigh more than $100 \mathrm{~kg} / \mathrm{m}^{2}$.

The new pharmacy robot is conceptually very simple. That reason, and thanks of its modularity, allows a quick and easy maintenance and any breakdown can be easily repaired.

As explained above, the technology used in this new generation of robots is based on new smart devices, called micro-servers, which provide the system with the optimal decentralization, avoiding the typical collapse that centralized systems suffer sometimes. Thanks to this decentralization, the new generation of robots is able to deliver so many products as different kind of products have stored simultaneously, becoming on the fastest dispensing robot in the market. Meanwhile the classical solutions can only dispense one product simultaneously. From another point of view, a classical solution takes between $2.5 \mathrm{~s}$ and $3 \mathrm{~s}$ to dispense one product, which means that it takes at least 25 $\mathrm{s}$ to dispense 10 products. The new architecture allows the system to dispense one product of each kind that it stores, in $2 \mathrm{~s}$.

Security is an issue that has been taken into account from the outset when it began to design this new generation of robots; for this reason, the system is based on micro-electronic systems allowing the safe interaction with users. In contrast, the classical solutions are based on powerful manipulators, requiring that the system provide a safety enclosure to prevent the access to users.

This is a product that will be facing the public in pharmacies, so an attractive and customizable design is fundamental to improve the image of the business where it is installed. In this way, the robot not only improves the performance and business benefits, but also will make it more attractive for the customer.

There are a wide range of pharmacies, so the new generation of pharmacy robots is scalable, ie, the basic range has automatic dispensing and semi-automatic replenishment; however, the robot is ready to install the automatic repositioning system.

Last but not least important, is the ecological aspect. The new generation of robots consumes less than $60 \mathrm{~W}$ (similar power that a single bulb consumes), compared to more than $2 \mathrm{~kW}$ consumed by the classical solutions.

All these above characteristics give great value to this new solution and they make this a new generation of pharmacy robots (see Table 1).

\section{CONCLUSIONS}

This paper presents a revolutionary pharmacy robot based on industrialized micro-servers and web 2.0 technologies. This new robot, thanks to its architecture, provides the system with speed, local intelligence, remote control and more efficiency.

It's able to dispense 250 products simultaneously. The number of dispensed products at the same time depends on the robots configuration. So, this robot is the fastest of the market.

This system is totally noiseless because its power supply is only electronic. It is robust, modular and reliable due to the union between this robot and this kind of architecture.

We have built a prototype and its results were very successfully. The firm Cenker Robotics S.L has acquired the patent rights of this novel technology based on the microservers with the mechanical system, so now, Cenker is marketing this technology, installing the new generation of pharmaceutical robots.

TABLE I. COMPARATIVE TABLE

\begin{tabular}{|c|c|c|}
\hline Item & Cenker New Generation & Classic Solution \\
\hline Installation & Simple and easy & Difficult \\
\hline Maintenance & Fast, easy and cheap. & Difficult and expensive \\
\hline Technology & Decentralized (micro-servers) & Total, the user can't Access to the system \\
\hline Safety & Total, the user can Access to the system. & No \\
\hline Customizable & Yes & product / manipulator (simultaneously) \\
\hline Dispensing Speed & $<250$ products / robot (simultaneously) \\
\hline Ecologic (consume W) & Yes, customizable & Industrial \\
\hline Attractive & Yes & No \\
\hline Scalability & Yes & No \\
\hline Versatile & &
\end{tabular}




\section{REFERENCES}

[1] S.Collins, "Robot system becomes future on pharmacy at NHS Trust”, Assured media solutions, www.healthcareitnews.com

[2] Correo Farmaceútico: www.correofarmaceútico.com

[3] MP.Lee, "Automation and the future practice of pharmacy changing the focus of pharmacy", in press

[4] P.Troiano, "Automating the medication distribution process", in press

[5] Willach Pharmacy Solutions: http://www.willachheise.com/au/our-customers/index.php

[6] ThosemGroup Inc. "White papers on robotics", in press.

[7] J.Governor, D. Hinchcliffe and D. Nickull, "Web 2.0 Architectures”, O’Reilly Media, Inc, 2009

[8] K.Ogata, "Modern control engineering", $3^{\text {th }}$ ed.,Prentice Hall, 1990

[9] C.Marietti, "Robots hooked on drugs. Robotic automation explands pharmacy services", in press

[10] Rowa Automatisierungssysteme GmbH \& Co. KG - Alemania: www.rowa.de
[11] Tecny-Farma S.A. - España: www.tecnyfarma.com

[12] Match4: http://www.apotheka.com/

[13] Siemes Microserver: http://cn.siemens.com/cms/cn/English/SBT/ downloading/Building_Automation_Product_Home/Direct_ Digital_Controller/Documents/AEM200/149802_Rev3_EN 1173018.pdf

[14] WebLab-Deusto: https://www.weblab.deusto.es/joomla/

\section{AUTHORS}

J.García Zubia, is with the University of Deusto, Avd. Universidades 24, 48007 Bilbao (Spain) (e-mail: zubia@deusto.es).

I. Trueba Parra, is with the University of Deusto, Avd. Universidades 24, 48007 Bilbao (Spain) (e-mail: itrueba@eside.deusto.es).

This article was modified from a presentation at the REV2010 Conference at KTH, Stockholm, Sweden in June 2010. Submitted July 15th, 2010. Published as resubmitted by the authors July 29th, 2010. 